\title{
Modified Fiber Optic System for Monitoring the Railgun Rail Deflections
}

\author{
V. KLEIZA ${ }^{a, *}$ AND J. VERKELIS ${ }^{b}$ \\ ${ }^{a}$ Kaunas University of Technology, Panevėžys Institute, Klaipėdos str. 1, LT-35209 Panevėžys, Lithuania \\ ${ }^{b}$ Center for Physical Sciences and Technology, Semiconductor Physics Institute
}

A. Goštauto str. 11, LT-01108 Vilnius, Lithuania

The work presents a research into adequacy of two models of an individual fiber optic sensor of a system of identical sensors, which is designed for measuring the railgun rail deflections. The presented models are compared with the data of an experiment. It has been found that a more simple model (in view of the scope of calculation) is more convenient as it concerns the calibration on the grounds of the data of the experiment.

PACS: $42.81 . \mathrm{Wg}$

\section{Introduction}

The objective of this investigation is to explore noncontact fiber-optic system for monitoring of the railgun rail deflections [1] (Fig. 1). Fiber optic sensors are well

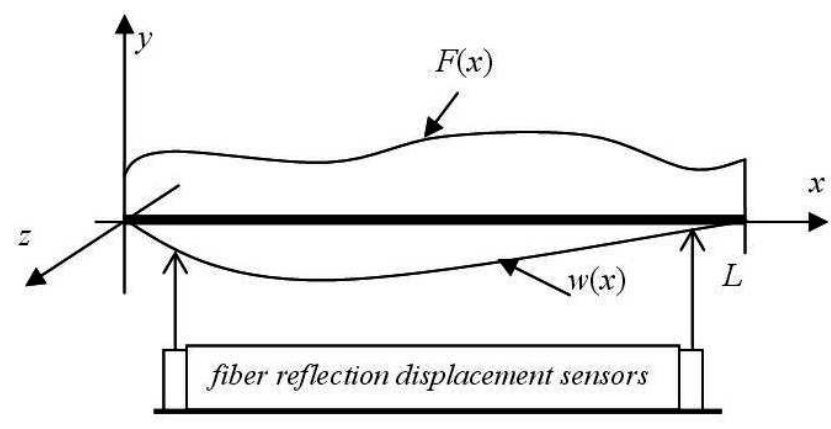

Fig. 1. Measurement scheme of the deflection of a beam. $F(x)$ is the transversely distributed load, $w(x)=$ $w\left(x_{i}\right)=w_{i}$ is experimental data obtained by beam deflection measurement by optical fiber reflection non-contact displacement sensors.

suited for that. They can be installed over many meters and are able to measure distributed strain and further physical and mechanical quantities with high resolution [2]. Furthermore, optical fibers can work in strong electromagnetic fields. The article is aimed at choosing a mathematical model of the distance sensor and providing grounds for its adequacy according to the experimental data. The sensor consists of a light transmitting fiber $(\mathrm{TF})$ and an identical receiving fiber $(\mathrm{RF})$ that de-

* corresponding author; e-mail: vytautas.kleiza@ktu.lt tects the light reflected power from the surface (mirror) (Fig. 2). Obtained results are reviewed and discussed from a model adequacy point of view. The study provides a fundamental understanding for developing of the mathematical model.

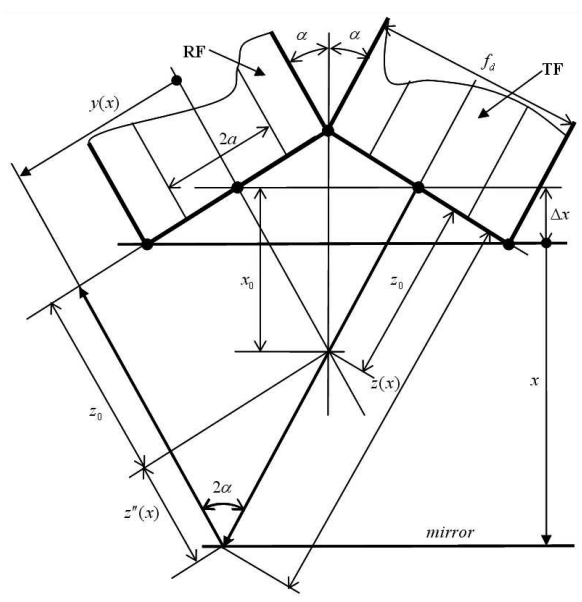

Fig. 2. Arrangement of fiber tips in the reflection fiber sensor (for the first model). TF is light transmitting fiber, $\mathrm{RF}$ is light receiving fiber; $2 a$ is the diameter of the fiber cladding, $f_{d}$ is the diameter of the fiber core.

\section{Mathematical models}

In the development of models of distance reflection fiber optic sensors two types of the mathematical models are used most often. The first one [3, 4] is based on calculation of power $P_{I}(x)$ according to a simple expression (see Fig. 2) 


$$
P_{I}(x)=\left[C_{0} \exp \left(-y^{2}(x) / R^{2}(x)\right)\right] / \pi R^{2}(x),
$$

where $x$ is distance to be measured; while TF and RF: $f_{d}$ - diameter of cladding, $2 a$ - diameter of core, $\theta_{a}$ acceptance angle, and $2 \alpha$ - angle between $\mathrm{TF}$ and $\mathrm{RF}$ axes. Moreover

$$
\begin{aligned}
& y(x)=z^{\prime \prime}(x) \tan (2 \alpha), \quad R(x)=a+z_{\rho}(x) \tan \left(\theta_{a}\right), \\
& z_{\rho}(x)=z(x)+z^{\prime \prime}(x)+z_{0}, \quad z(x)=(x+\Delta x) / \cos \alpha, \\
& x_{0}=\Delta x / \tan ^{2} \alpha .
\end{aligned}
$$

The second model is based on the calculation of far more complex integral [5-7] (Fig. 3). If the power $P_{0}$ which is generated by TF, in the event that one chooses the Gaussian intensity of radiation (among the possible intensities: constant, parabolic and Gaussian), then the intensity of radiation on the entire plane $\Pi$ (Fig. 3b), the origin of which is at the point $O\left(x, a, f_{d}, \alpha, \theta_{a}\right)$ (coordinates $y$ and $z$ ), equals to

$$
I\left(x, y, z, a, f_{d}, \alpha, \theta_{a}\right)=\frac{\exp \left(-\frac{y^{2}+z^{2}}{q^{2}\left(x, a, f_{d}, \alpha, \theta_{a}\right)}\right)}{\pi q^{2}\left(x, a, f_{d}, \alpha, \theta_{a}\right)},
$$

besides

$$
\frac{\iint_{S(\Pi)} \exp \left(-\frac{y^{2}+z^{2}}{q^{2}\left(x, a, f_{d}, \alpha, \theta\right)}\right) \mathrm{d} y \mathrm{~d} z}{\pi q^{2}\left(x, a, f_{d}, \alpha, \theta\right)}=P_{0},
$$

where $S(\Pi)$ is the entire plane $\Pi$.
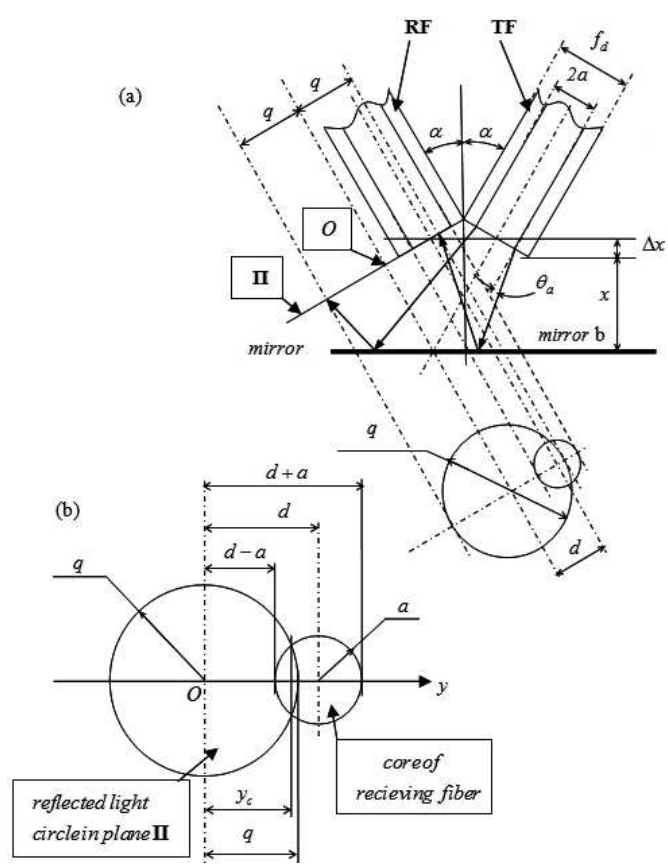

Fig. 3. (a) Arrangement of fiber tips in the reflection fiber sensor (for the second model). TF is light transmitting fiber, $\mathrm{RF}$ is light receiving fiber; $2 a$ is the diameter of the fiber cladding, $f_{d}$ is the diameter of the fiber core, and $\theta_{a}$ is acceptance angle. (b) Image of reflected light circle and core of $\mathrm{RF}$ at plane $\Pi$.
A part of the whole TF power $P_{0}$ which enters to the RF equals to

$$
\begin{aligned}
& P_{I I}\left(x, a, f_{d}, \alpha, \theta\right) \\
& \quad=\frac{\iint_{S\left(x, a, f_{d}, \alpha, \theta\right)} \exp \left(-\frac{y^{2}+z^{2}}{q^{2}\left(x, a, f_{d}, \alpha, \theta\right)}\right) \mathrm{d} y \mathrm{~d} z}{\pi q^{2}\left(x, a, f_{d}, \alpha, \theta\right)},
\end{aligned}
$$

where $S\left(x, a, f_{d}, \alpha, \theta\right)=S_{q} \bigcap S_{d}$ is the intersection of TF core image on the plane $\Pi$ and the RF core, and $S_{q}$ - image of TR core (circle), $S_{d}-\mathrm{RF}$ core (circle). Then the trigonometric relationships lead to the following equalities:

$$
\begin{aligned}
& S_{q}\left(x, a, f_{d}, \alpha, \theta_{a}\right) \\
& \quad=\left\{(y, z): y^{2}+z^{2} \leq q^{2}\left(x, a, f_{d}, \alpha, \theta_{a}\right)\right\} \\
& S_{d}\left(x, f_{d}, \alpha\right) \\
& \quad=\left\{(y, z):\left[y-d\left(x, a, f_{d}, \alpha, \theta_{a}\right)\right]^{2}+z^{2} \leq a^{2}\right\} \\
& q\left(x, a, f_{d}, \alpha, \theta_{a}\right)=a+\left(2 x \cos \alpha+f_{d} \sin 2 \alpha\right) \tan \theta_{a}, \\
& d\left(x, f_{d}, \alpha\right)=f_{d} \cos 2 \alpha-2 x \sin \alpha .
\end{aligned}
$$

The article examines the adequacy of the first and the second model, peculiarities of calculation, algorithms and their accuracy.

\section{Discussion}

The work presents examination of adequacy of both models (experimental setup is as in work [8]). In order to compare adequacy of the first (1) and the second (2) model the output signals and data of experiments were normalized, i.e. the following values were examined:

$$
\begin{aligned}
& P_{N I}=P_{I} / \max P_{I}, P_{N I I}=P_{I I} / \max P_{I I}, \\
& P_{N E}=P_{E} / \max P_{E},
\end{aligned}
$$

where $P_{E}$ - results of the experiment. Results of the calculation (Fig. 4) proved that more complex and more time-consuming in respect of calculation time is the second model (2), while its accuracy is similar to that of the first model (1). It should be noted that model (2) defines two dead zones (see Fig. 4, lines 4 and 5), however they are located in the intervals $x$ of the distances that are of low importance in practice.

Certain variance of modeling data and experiment data (Fig. 4) may result from the technique of the experiment, namely the angle $\alpha$ and, the most important, non-perpendicularity of the plane of the transmitting fiber tip in relation to the fiber axis. In our experiment we found that the angle of the plane of the transmitting fiber tip in relation to the fiber axis is 83 degrees (must be 90 degrees). When applying the first model this shortcoming may be easily introduced into the expression (1), 


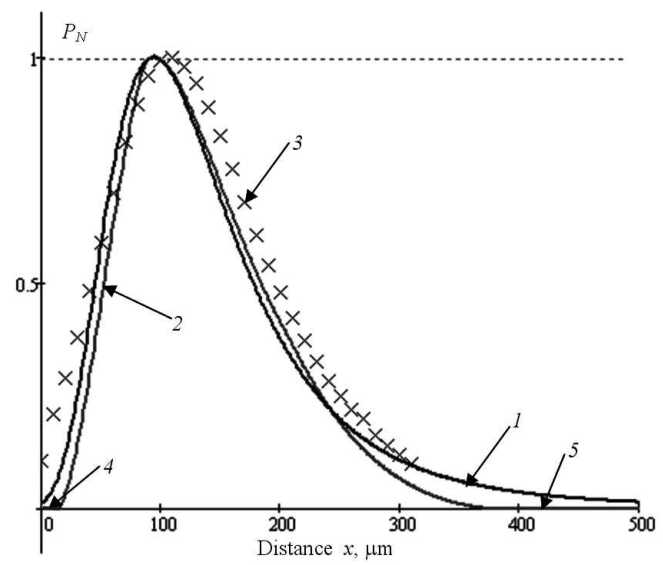

Fig. 4. Modeled (1 - first model, 2 - second model, 4, 5 - dead zones) and 3 - observed results. $a=$ $52.5 \mu \mathrm{m}, f_{d}=125 \mu \mathrm{m}, \alpha=30 \mathrm{deg}, \theta_{a}=12.709 \mathrm{deg}$.

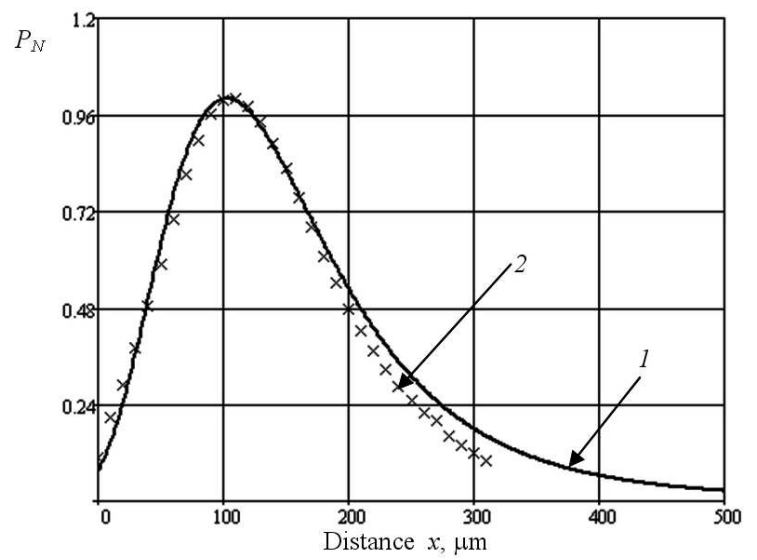

Fig. 5. Modeled (1 - first calibrated model) and $2-$ observed results. $P_{N}$ is normalized power, $a=52.5 \mu \mathrm{m}$, $f_{d}=125 \mu \mathrm{m}, \alpha=30 \mathrm{deg}, \theta_{a}=12.709 \mathrm{deg}$. i.e. the first model may be calibrated. The obtained results of calibrated model are presented in Fig. 5. We shall note that it is far more difficult and sometimes even not possible to calibrate the model (2) in the same manner.

\section{Conclusions}

The work presents a research into adequacy of two models of an individual fiber optic sensor of a system of identical sensors, which is designed for measuring the railgun rail deflections.

The presented models are compared with the data of an experiment.

It has been found that a more simple model (1) (in view of the scope of calculation) is more convenient as concerns the calibration on the grounds of the data of experiment. Calibration of the model (1) and comparison of the data with the data obtained during the experiment is made.

\section{References}

[1] J. Bareisis, V. Kleiza, in: Book of Abstracts of XVI Int. Conf. "Mechanics of Composite Materials-2010", Riga, 2010, p. 48.

[2] V. Kleiza, J. Verkelis, J. Vibroeng. 10, 180 (2008).

[3] Y. Libo, P. Jian, Y. Tao, M. Guochen, Sensors Actuators A 36, 177 (1993).

[4] Y.W.H. Ko, K.-M. Chang, G.-J. Hwang, Sensors Actuators A 49, 51 (1995).

[5] H. Huang, U. Tata, Appl. Opt. 47, 1302 (2008).

[6] P.B. Buchade, A.D. Shaligram, Sensors Actuators A 128, 312 (2006).

[7] P.B. Buchade, A.D. Shaligram, Sensors Actuators A 136, 199 (2007)

[8] V. Kleiza, J. Verkelis, Acta Phys. Pol. A 113, 1025 (2008). 